\title{
Génesis Instrumental del Concepto de Recta Tangente
}

\author{
Abilio Orts \\ Francisco José Boigues \\ Salvador Llinares
}

\begin{abstract}
RESUMEN
El objetivo de esta investigación es identificar características del proceso de génesis instrumental en el aprendizaje del concepto de recta tangente a una curva cuando se usa GeoGebra como recurso tecnológico. Diseñamos un experimento de enseñanza con 11 estudiantes de primer curso de educación pos-obligatoria (16-17 años) considerando una descomposición genética del concepto de recta tangente. El análisis de los procesos de resolución de los problemas nos ha permitido identificar tres características de la génesis instrumental: (i) el uso de la aproximación lineal a una curva, (ii) la mediación semiótica, y (iii) la coordinación de las concepciones cartesiana y leibniziana de la recta tangente. Estas características ayudan a explicar la relación entre los esquemas de uso (uso de los comandos del programa-artefacto) y los esquemas de acción instrumental (uso de los significados del concepto) generados durante la resolución de los problemas.

Palabras clave: Génesis Instrumental, Recta tangente, Aprendizaje matemático, Herramientas tecnológicas.
\end{abstract}

\section{Instrumental Genesis of the Concept of Tangent Line}

\section{ABSTRACT}

This research study has as goal to identify features of Instrumental Genesis in the learning of tangent line concept using GeoGebra. We design a teaching experiment with 11 high school students (16-17 years-old) considering a Genetic Decomposition of the tangent line concept. We have identify three features of high students' instrumental genesis: (i) the use of lineal approximation to a function, (ii) the semiotic mediation, and (iii) the coordination between Cartesian and Leibniz conceptions of tangent line. These features help us to explain the relation between the instrumented action schemes (taking account the operative invariants) and usage schemes (use of commands of technological resource) during the solving problem. tools.

Keywords: Genesis Instrumental, Tangent line, Mathematical learning, Technological

\footnotetext{
Abilio Orts es graduado en Matemáticas y Doctor en Investigación Educativa. Actualmente es profesor de Matemáticas en el IES Tavernes Blanques en Valencia. Dirección postal: IES Tavernes Blanques, C/Alacant, 14, 46016 Tavernes Blanques (Valencia), España. E-mail: abilioorts@gmail.com.

Francisco José Boigues es graduado en Matemáticas y Doctor en Ciencias de la Educación. Actualmente es profesor del departamento de Matemática Aplicada de la Universidad Politécnica de Valencia, Campus de Gandia, C/ Paraninfo 1, 46730 Grau de Gandia (Valencia), España. E-mail: fraboipl@mat.upv.es

Salvador Llinares es graduado en Matemáticas y Doctor en Ciencias de la Educación. Actualmente es catedrático de Didáctica de la Matemática en la Universidad de Alicante, España. Campus de San Vicente del Raspeig, E-03080 Alicante, España. E-mail: sllinares@ua.es

Recebido para publicação em 14 fev. 2018. Aceito, após revisão, em 02 abr. 2018
}

Acta Scientiae

Canoas

v. 20

n.2

p.78-95 mar./abr. 2018 


\section{INTRODUCCIÓN}

La tecnología se está incorporando al aula de matemáticas y forma parte del entorno de aprendizaje de los estudiantes (Faggiano, Ferrara y Montone, 2017; NCTM, 2015). Esta situación genera cuestiones sobre el papel que desempeñan los recursos tecnológicos en el proceso de enseñanza-aprendizaje de las matemáticas (Alqahtani y Powell, 2016, 2017; Boigues, Estruch y Llinares, 2010). Por ejemplo, el uso de un software de geometría dinámica como el GeoGebra, permite a los estudiantes explorar los objetos matemáticos, realizar conjeturas, validarlas y justificar sus resultados (Artigue, 2002; Arzarello, Olivero, Paola y Robutti, 2002, Stahl, 2015; Orts, Llinares y Boigues, 2016a). Además, los recursos tecnológicos son instrumentos de mediación semiótica gracias a su potencialidad para presentar simultáneamente varias representaciones de un mismo objeto matemático (Lagrange y Artigue, 2009; Maschietto, 2008; Drijvers y Trouche, 2008). En esta investigación nos planteamos estudiar el papel que puede desempeñar el uso de un recurso tecnológico como GeoGebra en el aprendizaje de conceptos de Análisis Matemático en la educación pos-obligatoria (16-17 años). En particular el concepto de recta tangente a la gráfica de una función en un punto.

En el currículo español, los estudiantes acceden por primera vez al concepto de recta tangente durante el primer curso de la Educación Secundaria Obligatoria (12 años) y lo hacen desde un punto de vista geométrico. En ese momento se les define a través de un caso particular, la recta tangente a la circunferencia es la recta que toca a la circunferencia en un solo punto, y consecuentemente la recta rozaría a la circunferencia sin atravesarla. Es decir, la recta tangente es aquella recta que toca la circunferencia pero no la corta (concepción euclídea). Cuando los estudiantes llegan al primer año de educación pos-obligatoria (16 años) se les define la recta tangente a la gráfica de cualquier función desde el punto de vista del Análisis Matemático como la posición límite de las rectas secantes (que refleja una concepción cartesiana). Este planteamiento curricular implica un cambio desde la concepción euclídea estudiada en el primer año de la educación obligatoria (12 años) a una concepción analítica introducida en el primer año de la educación pos-obligatoria (16 años). Este cambio genera dificultades en los estudiantes (Orts, 2017). En esta situación, se plantean cuestiones relativas a si los recursos tecnológicos, y en particular el software GeoGebra, pueden ayudar a favorecer la transición desde la concepción euclídea a la cartesiana del concepto de recta tangente a una curva.

\section{MARCO TEÓRICO: LA GÉNESIS INSTRUMENTAL}

La génesis instrumental conjuga aspectos cognitivos y antropológicos que pueden ser usados para explicar los procesos de aprendizaje de los estudiantes cuando usan recursos tecnológicos al resolver problemas (Artigue, 2007). Uno de los puntos básicos de esta teoría es la diferencia entre artefacto e instrumento. El artefacto es la herramienta propiamente dicha, mientras que hablamos de instrumento cuando el usuario establece una relación significativa con la tarea a realizar a través del instrumento (Verillon y Rabardel, 1995). Es decir, el artefacto es dado, mientras que el instrumento es construido por el 
usuario a través de la actividad realizada. Desde este punto de vista, el uso de un artefacto puede mejorar las habilidades de un estudiante, si bien esto puede afectar a cuestiones de carácter conceptual que deben examinarse.

El proceso por el que un artefacto se convierte en instrumento se denomina génesis instrumental (Drijvers, Kieran y Mariotti, 2010). La génesis instrumental consiste en la formación de esquemas instrumentales (que pueden ser de uso o de acción instrumental) entendidos como formas estables de tratar determinadas tareas por parte del estudiante. En la génesis instrumental podemos observar dos procesos. El primero, denominado instrumentalización, se dirige del sujeto hacia al artefacto y en él se desarrollan esquemas de uso, relacionados con la manipulación técnica del artefacto. Esquemas de uso son, por ejemplo, encender un ordenador, ajustar el contraste de la pantalla, elegir una tecla determinada de una calculadora o saber usar el zoom en GeoGebra (Trouche, 2004). Con el segundo proceso, llamado instrumentación, el sujeto desarrolla esquemas de acción instrumental, que son aquellos relacionados directamente con el uso del artefacto con vistas a realizar una tarea. Es decir, estos esquemas permiten al estudiante entender las potencialidades y restricciones del artefacto para la resolución de una actividad matemática y se constituyen progresivamente en técnicas que permiten una respuesta efectiva al reconocer invariantes en la resolución de las tareas (uso del conocimiento mediado por el artefacto). Un ejemplo de esquema de acción instrumental es el cálculo del límite de una función (Trouche, 2004). Aquí, los esquemas de uso están dirigidos al manejo del artefacto mientras que los esquemas de acción instrumental son los que ponen en juego los significados del concepto de límite que permiten resolver la actividad. Para que un artefacto se convierta en instrumento, el estudiante debe apropiarse del artefacto a través del proceso de instrumentalización. Para ello, los estudiantes necesitan adquirir esquemas instrumentales que les van a permitir resolver problemas.

La génesis instrumental teoriza la relación entre los estudiantes y los instrumentos cuando resuelven tareas matemáticas. Es decir, cómo los estudiantes interactúan con los artefactos que median su actividad matemática durante la resolución de un problema llegando a convertirlos en instrumentos. Rabardel y Beguin (2005) subrayan que el instrumento es una entidad mixta nacida tanto del usuario como del artefacto. Es decir, el instrumento es un ente doble, parte correspondiente al artefacto y parte psicológica como utilización de esquemas (Alqahtani y Powell, 2016).

Es importante señalar que la utilización de un artefacto no es neutral para el sujeto pues el artefacto introduce en el sujeto una actividad cognitiva de construcción o de evolución de esquemas instrumentales (Rabardel, 1995). La estructura y la funcionalidad de los artefactos configuran la forma en que un alumno utiliza dicho artefacto, lo que da lugar a la formación del pensamiento del estudiante. Por otro lado, las interacciones de los estudiantes con el artefacto también configuran dicho artefacto y cómo es usado (Alqahtani y Powell, 2016).

Guin y Trouche (1999) muestran las diferencias en los procesos de instrumentación en función de la complejidad del entorno tecnológico (software) utilizado. Cuanto más complejo es el artefacto más simple parece la actividad, pues en la mayoría de ocasiones, 
esta se reduce a aplicar un solo comando en el que el proceso interno queda enmascarado y se hace más difícil para el estudiante realizar un control eficiente del instrumento. Por tanto, la elección del artefacto que proponemos a los estudiantes es un factor crucial para el desarrollo de la génesis instrumental.

Desde estas referencias nos planteamos identificar características del proceso de génesis instrumental en el aprendizaje del concepto de recta tangente a una curva cuando se usan recursos tecnológicos que favorecen la transición desde una concepción euclídea a una concepción cartesiana.

\section{MÉTODO}

\section{Participantes}

Once estudiantes de primer curso de educación pos-obligatoria (16-17 años) organizados en cuatro parejas y un grupo de tres. Cada grupo de estudiantes resolvía las tareas planteadas con un ordenador. Estos estudiantes poseen inicialmente una concepción geométrica global de recta tangente, asociada a una circunferencia, a la que hemos denominado concepción euclídea. El objetivo del experimento de enseñanza era que los estudiantes construyeran, a partir de la reflexión sobre los efectos producidos por las actividades realizadas, una concepción local de recta tangente a la gráfica de una función, a la que hemos denominado concepción cartesiana. Para el tránsito desde la concepción euclídea a la cartesiana nos valemos de la linealidad local de una función en el entorno de un punto en el que la función es derivable (concepción leibniziana) ya que al prolongar el segmento en el que se encuentra el punto de tangencia obtenemos la recta tangente.

\section{Experimento de enseñanza}

Diseñamos un experimento de enseñanza para estudiantes de primer curso de educación pos-obligatoria (16 años) con el objetivo de ayudarles a superar el obstáculo cognitivo generado por la concepción euclídea de la recta tangente cuando se aplica a cualquier tipo de curva. El experimento de enseñanza se diseñó considerando una descomposición genética del concepto de recta tangente, que se apoya en la concepción leibniziana mediante la interiorización de la concepción cartesiana (Orts et al., 2016b).

Como recurso tecnológico usamos GeoGebra. Una de las características de GeoGebra es su facilidad de uso frente a otros asistentes ya que el uso de los comandos en GeoGebra es intuitivo. Por ejemplo, se puede escribir directamente la expresión de la función en la barra de entrada y esta aparece representada gráficamente. Otros esquemas de uso, como por ejemplo el zoom, se pueden seleccionar directamente del menú de la Vista Gráfica. Además, la posibilidad de compartir la vista gráfica y 
algebraica en la pantalla del ordenador facilita la conversión del lenguaje simbólico al gráfico potenciando la mediación semiótica en el proceso de construcción de significados del concepto. Los esquemas de uso están relacionados con todas aquellas acciones que el estudiante debe conocer acerca del software GeoGebra para poder utilizarlo, mientras que los esquemas de acción instrumental se refieren a las acciones que los alumnos realizan con un propósito determinado durante la resolución de un problema.

El experimento de enseñanza constaba de doce actividades que los estudiantes debían resolver en cuatro sesiones y fueron diseñadas con el objetivo de favorecer la construcción del concepto de recta tangente desde la concepción cartesiana. En nuestro experimento de enseñanza hemos definido la recta tangente a partir de la concepción leibniziana, ayudándonos del recurso del zoom de GeoGebra (Figura 1), para luego coordinar dicha concepción con la cartesiana con la ayuda de un deslizador (comprobando que la recta en la que la gráfica de la función se convierte al hacer zoom en el entorno del punto es la recta límite de las rectas secantes).
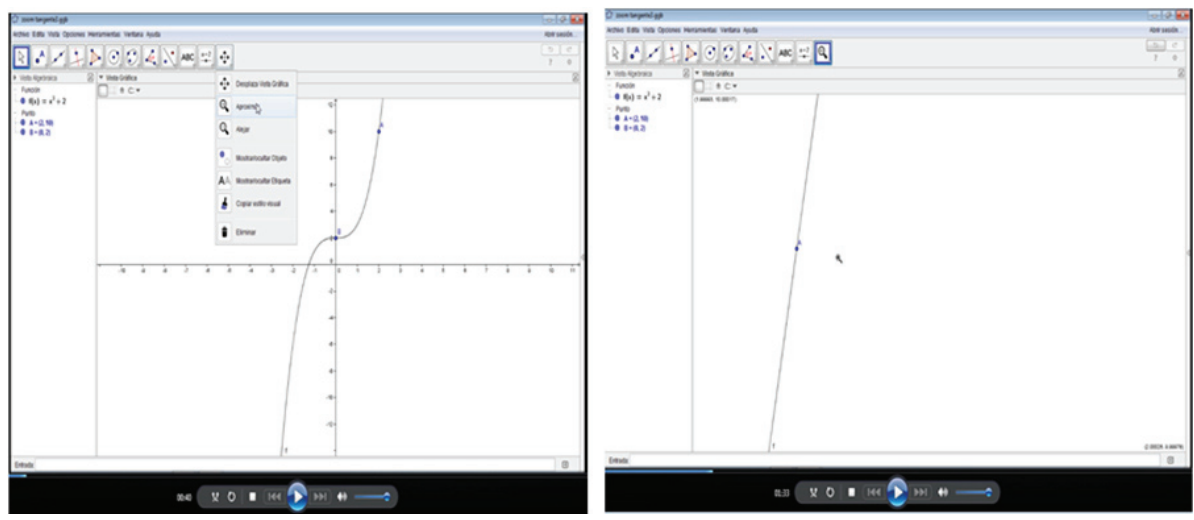

Figura 1. Uso del zoom de GeoGebra para comprobar la linealidad local de una función

Las tareas se diseñaron para que el uso del GeoGebra permitiera poner de manifiesto las limitaciones de la concepción euclídea del concepto de recta tangente con el que los estudiantes entran en el primero curso de la educación pos-obligatoria (Figura 2).

Las sesiones del experimento se grabaron con el programa CamStudio ${ }^{\circ}$. Este programa es un software instalado en el ordenador que con una web-cam registra las interacciones verbales de los estudiantes durante la resolución de las actividades y las manipulaciones que cada grupo realizaba con el ordenador, en concreto, las que realizaba con GeoGebra. 


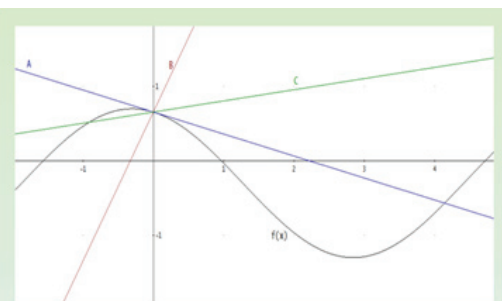

Act.1: Recta tangente a f en $x=0$

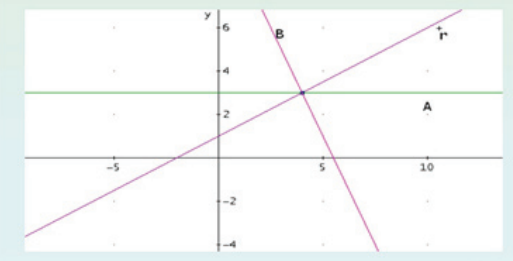

Act.3: Recta tangente a la recta $y=\frac{x}{2}+1$

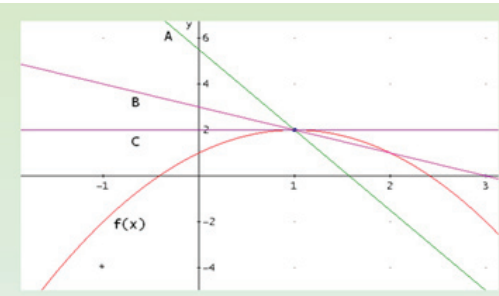

Act.2: Recta tangente a f en un máximo relativo

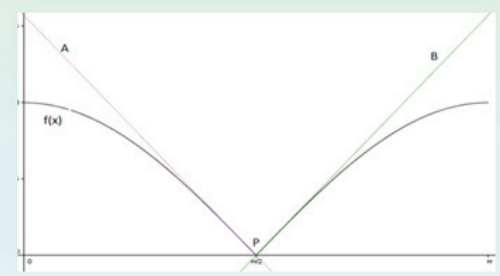

Act.4: Recta tangente en un punto anguloso

Figura 2. Actividades 1 a 4 de la sesión 1 cuya finalidad era mostrar las limitaciones de la concepción euclídea

\section{Análisis}

Después del experimento de enseñanza analizamos las respuestas de los cinco grupos de estudiantes a las doce tareas. Para atender a la génesis instrumental identificamos los esquemas de uso y de acción instrumental que se ponían de manifiesto durante la resolución de las tareas. Para ello analizamos todos los registros de los datos y nos fijamos en el papel que desempeñan los recursos tecnológicos en aquellos momentos en los que habíamos identificado una característica del aprendizaje conceptual dada por la descomposición genética del concepto de recta tangente entendida como un modelo de progresión en la construcción del concepto (Orts, 2017).

\section{RESULTADOS}

En esta sección presentamos tres características de la génesis instrumental identificadas en el aprendizaje del concepto de recta tangente a una curva cuando usamos GeoGebra para favorecer la transición desde una concepción euclídea a una concepción cartesiana:

el desarrollo del esquema de acción instrumental que genera la concepción leibniziana mediante el esquema de uso del zoom;

Esquema de uso de los modos de representación manifestados en la generación de esquemas de acción instrumental de mediación semiótica y las aproximaciones por tanteo, $\mathrm{y}$ 
Esquema de uso del deslizador en la generación del esquema de acción instrumental de coordinación de las concepciones cartesiana y leibniziana.

\section{Esquema de uso del zoom y esquema de acción instrumental que genera la concepción leibniziana del concepto de recta tangente}

La actividad 3 de la sesión 2 tenía como objetivo la interiorización de la concepción leibniziana.

Actividad 3 Sesión 2: Dada la curva $f(x)=2 x^{2}-x$, averigua cuál de las siguientes rectas es tangente a $f(x)$ en $\mathrm{x}=1$ : a) $y=3 x-2$; b) $y=\frac{17 x-12}{5}$

En la resolución de esta actividad, los estudiantes del grupo 4 usan el esquema de uso del zoom para comprobar qué ocurre con las gráficas de la función y de la recta tangente en el entorno de un punto donde la función es derivable. De esta manera, se puede evidenciar el esquema de acción instrumental que permite interiorizar la concepción leibniziana de la recta tangente para comprobar cómo en el punto en que la función es derivable, la gráfica de la función y la de la recta tangente se superponen (Figura 3).

Actividad 3 Sesión 2: Dada la curva, $f(x)=2 x^{2}-x$, averigua cuál de las siguientes rectas es tangente en $\mathrm{x}=1$ : a) $y=3 x-2$; b) $y=\frac{17 x-12}{5}$

Los estudiantes S y K, integrantes del grupo 4, representan gráficamente la función y las dos rectas $\mathrm{y}$ razonan del modo siguiente:

S: Están muy juntas una de la otra pero una será recta tangente y la otra no.

[realizan un zoom]

K: Es que se cruzan, ¿no?

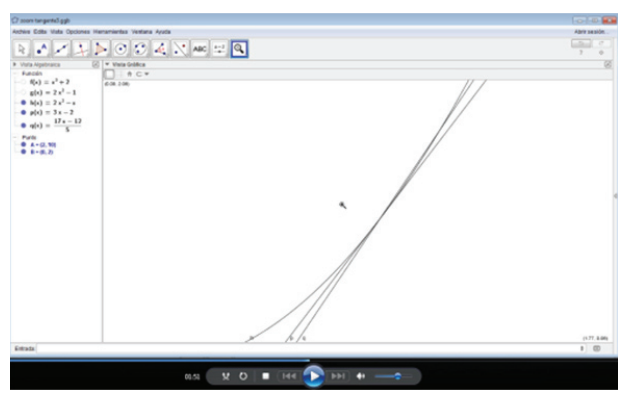


K: Mira podemos observar que en este punto prácticamente se juntan.

$\mathrm{S}$ : Vamos a intentar aproximarnos bien porque así se mezcla un poco todo.

$\mathrm{K}$ : Sí, porque se juntan las dos.

S: Se mezcla, no sé cuál es cuál.

[cambian el color de las rectas]

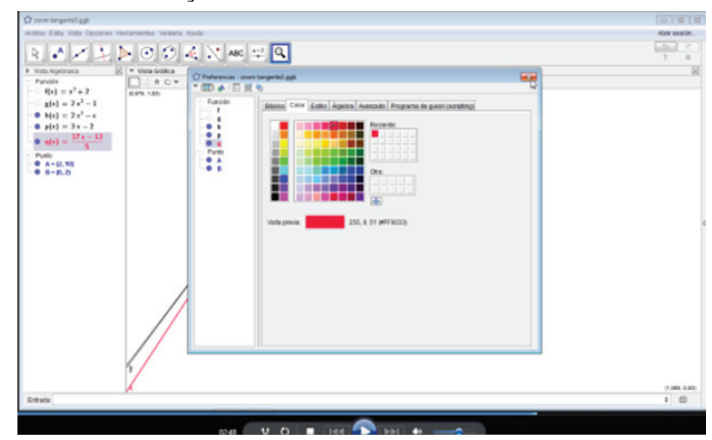

S: Vale ahora mejor. Sí, la curva y la recta verde son las que se confunden.

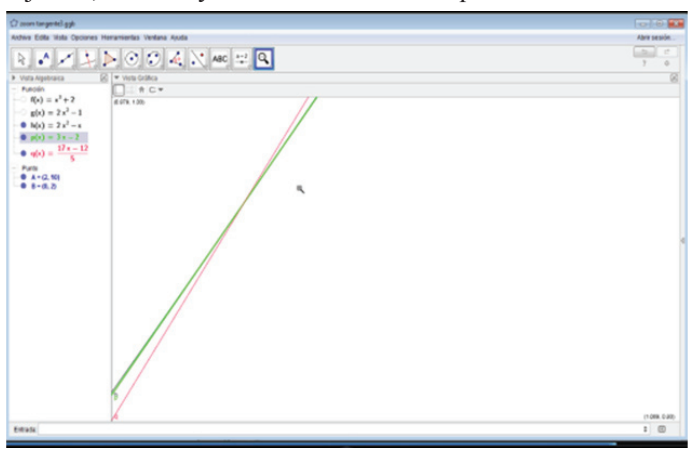

S: Parece que esté una sobre la otra, entonces esa es la tangente.

K: Sí.

$\mathrm{S}$ : Entonces es la opción a, y=3x-2.

$\mathrm{K}$ : Sí, es esa.

S: Vale.

K: Se confunden.

$\mathrm{S}$ : Sí, al no cambiar el color no lo veíamos bien porque se mezcla mucho...

K: Ya.

$\mathrm{S}:$... pero si nos fijamos, en el punto ese al aproximarnos lo hemos visto que se confunden mucho.

$\mathrm{K}$ : Una pasa encima de la otra, entonces esa tiene que ser la recta tangente.

S: Claro, entonces la recta tangente es esta.

Figura 3. Protocolo de actuación del grupo 4 en el que se muestra cómo han interiorizado la concepción leibniziana con la ayuda del zoom de GeoGebra 
Mientras que los estudiantes del grupo 1, aunque conocen el esquema de uso del zoom, no son capaces de crear el esquema de acción instrumental correspondiente que les permita visualizar la concepción leibniziana. Esto estudiantes no hacen los zooms necesarios para llegar a la superposición de las gráficas lo que evidencia que siguen anclados en una concepción euclídea de la recta tangente (la toca pero no la corta), si bien han cambiado su concepción global euclídea por la correspondiente versión local (Figura 4).

Actividad 1 Sesión 4: Obtén la ecuación de la recta tangente a la curva $f(x)=x^{2}$ en el punto de abscisa $\mathrm{x}=1$. Opciones: a) $\mathrm{x}=1$; b) $\mathrm{y}=2 \mathrm{x}-1$; c) $y=x-\frac{1}{2}$; d) ninguna de las anteriores.

Los estudiantes del grupo 1 representan gráficamente la función $f(x)=x^{2}$ y las candidatas a ser rectas tangente a la curva. A continuación concluyen, tras hacer un zoom, que la recta tangente es $\mathrm{y}=2 \mathrm{x}-1$ pues

"al ampliarla se ve que la toca pero no la corta; y las otras, pues la cortan".

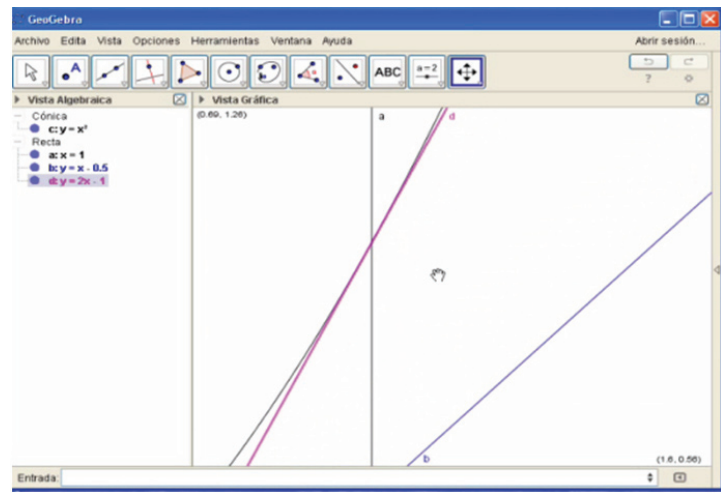

Figura 4. Protocolo de actuación del grupo 1 en el que se muestra cómo usan la concepción euclídea en modo local con la ayuda del zoom de GeoGebra

A partir de los protocolos en las Figuras 3 y 4, observamos cómo los estudiantes han desarrollado un esquema de acción instrumental de cambio de color de las gráficas. Por defecto, la versión de GeoGebra usada en el experimento representa todas las gráficas con idéntico color, por lo que no es sencillo identificar la ecuación de la función con su correspondiente gráfica. Esto lo solucionan los estudiantes cambiando el color de las gráficas (cada función se representa con un color diferente) para poder discriminar gráficamente cuál de las rectas propuestas es la recta tangente buscada.

\section{Esquema de uso de los modos de representación y el esquema de acción instrumental de mediación semiótica}

Otros esquemas de acción instrumental identificados son el uso de la representación gráfica de una función para coordinar los registros gráfico y analítico. Por ejemplo, los estudiantes del grupo 2 han resuelto la actividad 3 de la sesión 4 mediante un procedimiento analítico-algebraico y así obtienen que la recta tangente pedida tiene 
ecuación $y=-2 x+3,75$. A continuación, representan gráficamente la función inicial, la recta dada y la recta encontrada que debía ser, por un lado, tangente a la gráfica de la función y, por otro, paralela a la recta inicial. Con este esquema de acción instrumental, que usa la representación gráfica de funciones con GeoGebra, los estudiantes son capaces de coordinar los registros gráfico y analítico y así, comprobar en el registro gráfico la validez de la solución obtenida en el registro analítico (Figuras 5 y 6).

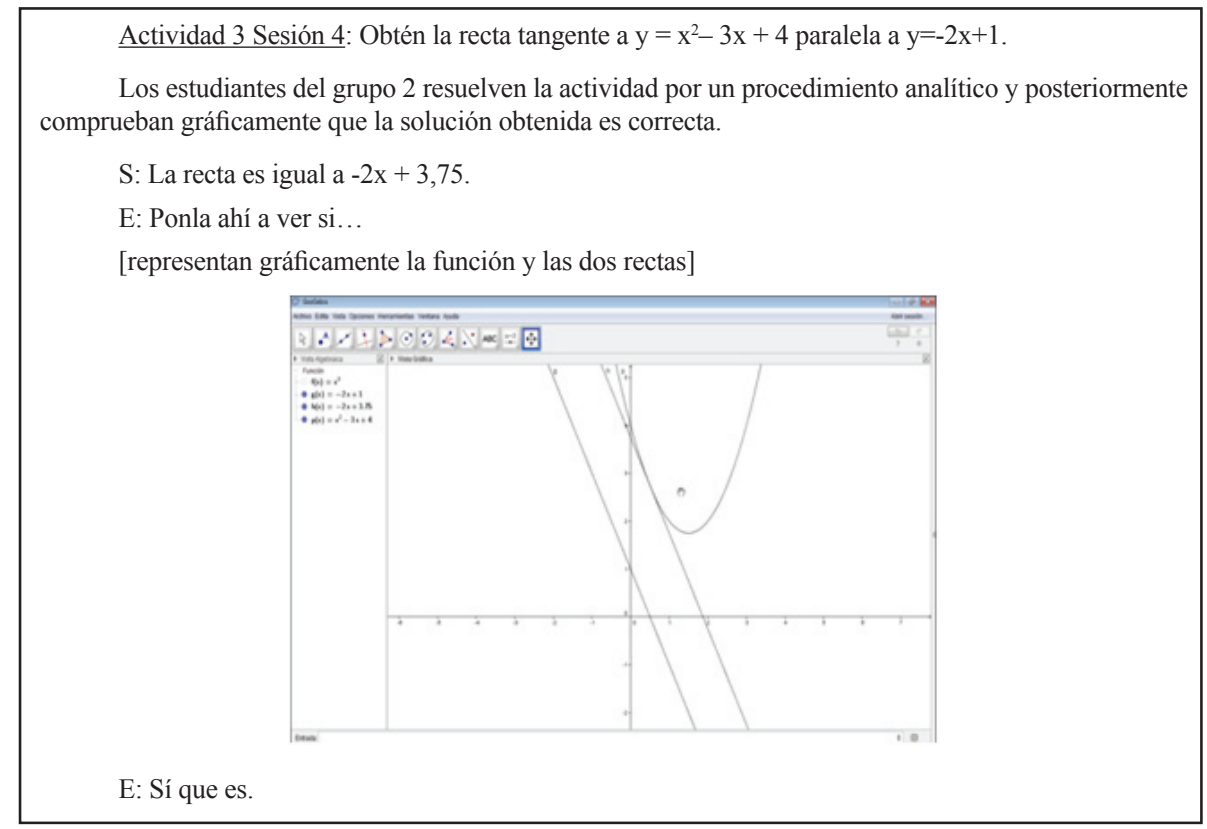

Figura 5. Protocolo de actuación del grupo 2 en el que se muestra cómo los estudiantes coordinan los registros gráfico y analítico con la ayuda de GeoGebra

\section{Esquema de uso de los modos de representación para resolver problemas por tanteo}

Otro ejemplo de este esquema de acción instrumental lo vemos en la manera en la que los estudiantes del grupo 5 resuelven la actividad 5 de la sesión 4:

Actividad 5 Sesión 4: Una determinada función $\mathrm{f}(\mathrm{x})$ pasa por el punto $(1,2)$. Además se sabe que su derivada en $x=1$ vale 3 , es decir, $f^{\prime}(1)=3$. Obtén de manera aproximada el valor de $f(1,027)$. Esboza un gráfico que explique tu respuesta.

En primer lugar los estudiantes calculan analíticamente la recta tangente a la gráfica de la función en el punto $\mathrm{x}=1$, obteniendo la recta $\mathrm{y}=3 \mathrm{x}-1$. A continuación, la utilizan para aproximar el valor de la función en un entorno del punto $(\mathrm{x}=1,027)$. Es decir, $\mathrm{y}=3 \cdot 1,027$ -1 , o sea, $\mathrm{y}=2,081$ con lo que concluyen que $\mathrm{f}(1,027) \approx 2,081$. 
A la hora de representar un gráfico que explique la situación, identifican la función original $\operatorname{con} f(x)=x^{3}+1$, es decir, realizan una integral sin conocer dicho concepto, buscando una función que pase por el punto $(1,2)$ y cuya derivada en $\mathrm{x}=1$ sea 3 .

Para comprobar gráficamente la solución analítica recurren al esquema de acción instrumental correspondiente. Así, representan gráficamente la función original obtenida y la recta, comprobando que la recta es tangente a la gráfica de la función en el punto $(1,2)$. Para ello se valen del zoom para desarrollar un esquema de acción instrumental que les permite ver que en el entorno del punto la gráfica de la recta y la de la función se superponen (Figura 6).

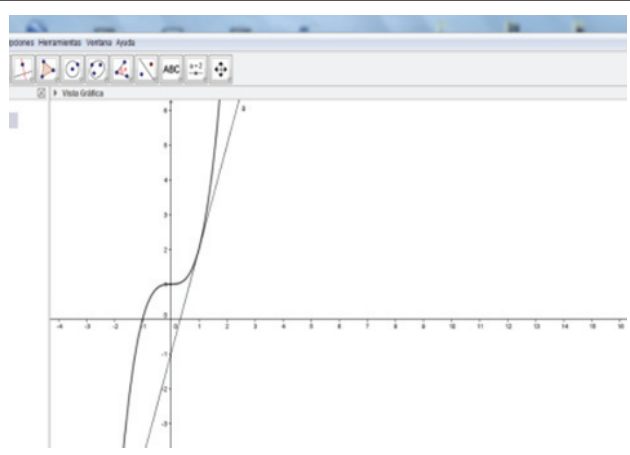

[realizan un zoom para acercar]
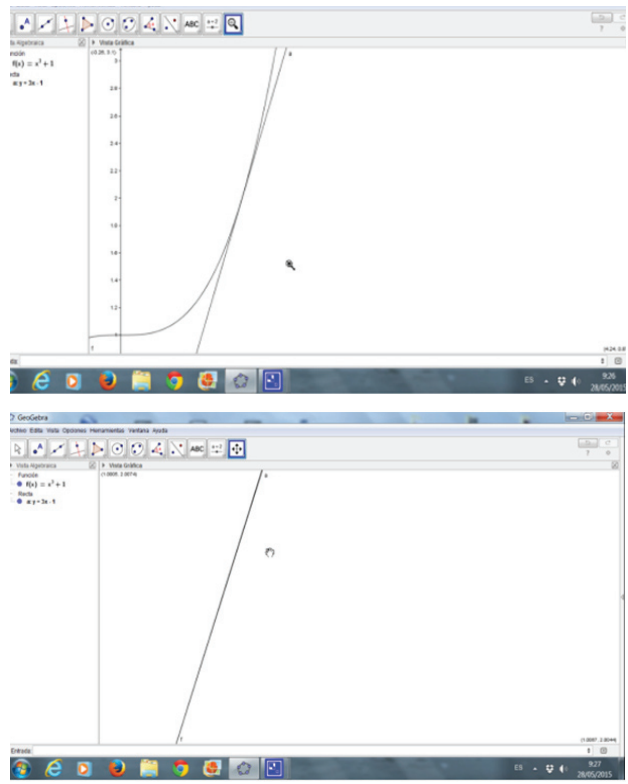

A: Está perfecto.

Figura 6. Protocolo de actuación del grupo 5 en el que se muestra cómo los estudiantes desarrollan un esquema de acción instrumental para coordinar los registros gráfico y analítico 
Un esquema de acción instrumental en el que combinan un método de tanteo numérico con la representación gráfica de funciones con GeoGebra es el que usan los estudiantes del grupo 1 para resolver la actividad 3 de la sesión 4:

Actividad 3 Sesión 4: Obtener la recta tangente a $y=x^{2}-3 x+4$ paralela a $y=-2 x+1$

Para obtener la ecuación de la recta tangente a la parábola dada y que sea paralela a la recta $y=-2 x+1$, los estudiantes recurren a un método gráfico de tanteo. Es decir, usan la representación gráfica de una función en GeoGebra como un esquema de uso que les permite resolver esta actividad por lo que evidencian que han generado un esquema de acción instrumental usando la forma analítica de una función afín $(\mathrm{y}=\mathrm{ax}+\mathrm{b})$.

Como estos estudiantes saben que la recta pedida será de la forma $\mathrm{y}=-2 \mathrm{x}+\mathrm{n}$ por ser paralela a la recta dada, prueban primero con la recta $y=-2 x+3$. Al representar conjuntamente en GeoGebra la parábola y la recta ven que esta no es la recta tangente que están buscando (Figura 7).

A continuación, prueban con la recta $y=-2 x+4$ y comprueban, de nuevo mediante la representación gráfica, que dicha recta tampoco es tangente a la gráfica de la función. Como ven que la recta buscada debe estar entre ambas rectas anteriores, prueban con una recta que tenga una ordenada en el origen entre los valores anteriores, es decir, entre $\mathrm{n}=3 \mathrm{y} \mathrm{n}=4$. Así, en este caso proponen como recta tangente la recta $\mathrm{y}=-$ $2 x+3,5$. De nuevo, y a partir de la representación gráfica comprueban que tampoco se trata de la recta buscada. Por último, dan con la solución probando con la recta $y=-2 x+3,75$. 


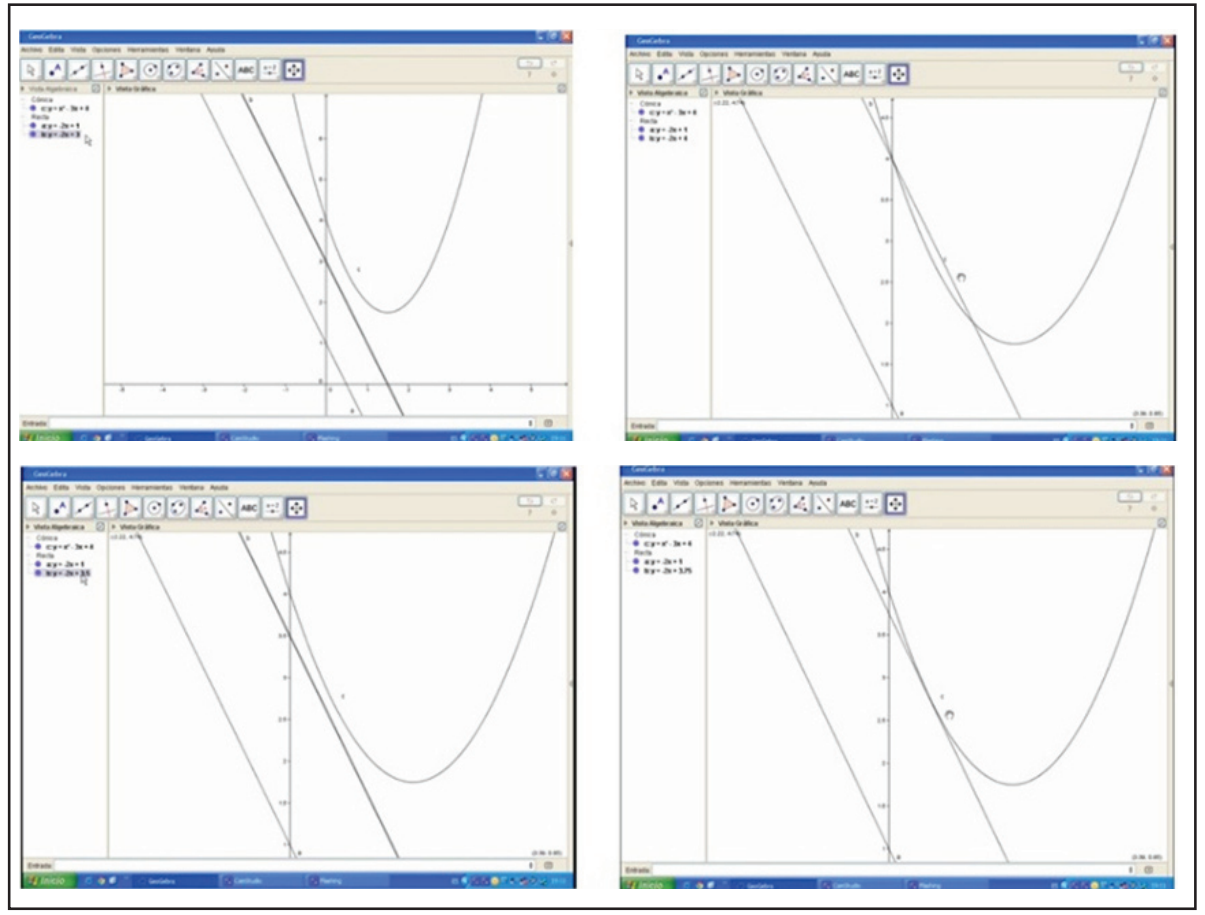

Figura 7. Protocolo de actuación del grupo 1 en el que se muestra cómo los estudiantes del grupo 1 utilizan un esquema de acción instrumental para resolver un problema por tanteo numérico con la ayuda de la representación gráfica de GeoGebra

\section{Esquema de uso del deslizador y el esquema de acción instrumental de coordinación de las concepciones cartesiana y leibniziana}

El uso del deslizador (esquema de uso) permitió a los estudiantes visualizar de una manera dinámica la convergencia de las rectas secantes a la recta tangente lo que permite asumir la generación de un esquema de acción instrumental de coordinación de las concepciones cartesiana y leibniziana. Por ejemplo, mediante un deslizador (definido como la distancia $h$ entre las abscisas del punto de tangencia y del otro punto que se considera para obtener la recta secante) se puede introducir la convergencia como un proceso dinámico. En la Figura 8 la recta tangente viene representada en color verde (los estudiantes comprueban que efectivamente es la recta tangente mediante sucesivos zooms verificando que en el entorno del punto de tangencia las gráficas de la recta tangente y la de la función se superponen). Variando el deslizador (en esta figura aparece inicialmente con valor $h=2$ ) se crean las condiciones para visualizar la convergencia de las rectas secantes a la recta tangente. Esta situación permite relacionar la tendencia de las pendientes de las 
rectas secantes con la derivada de la función en dicho punto visto a través de la recta a la que converge la sucesión de rectas secantes al mover el deslizador.

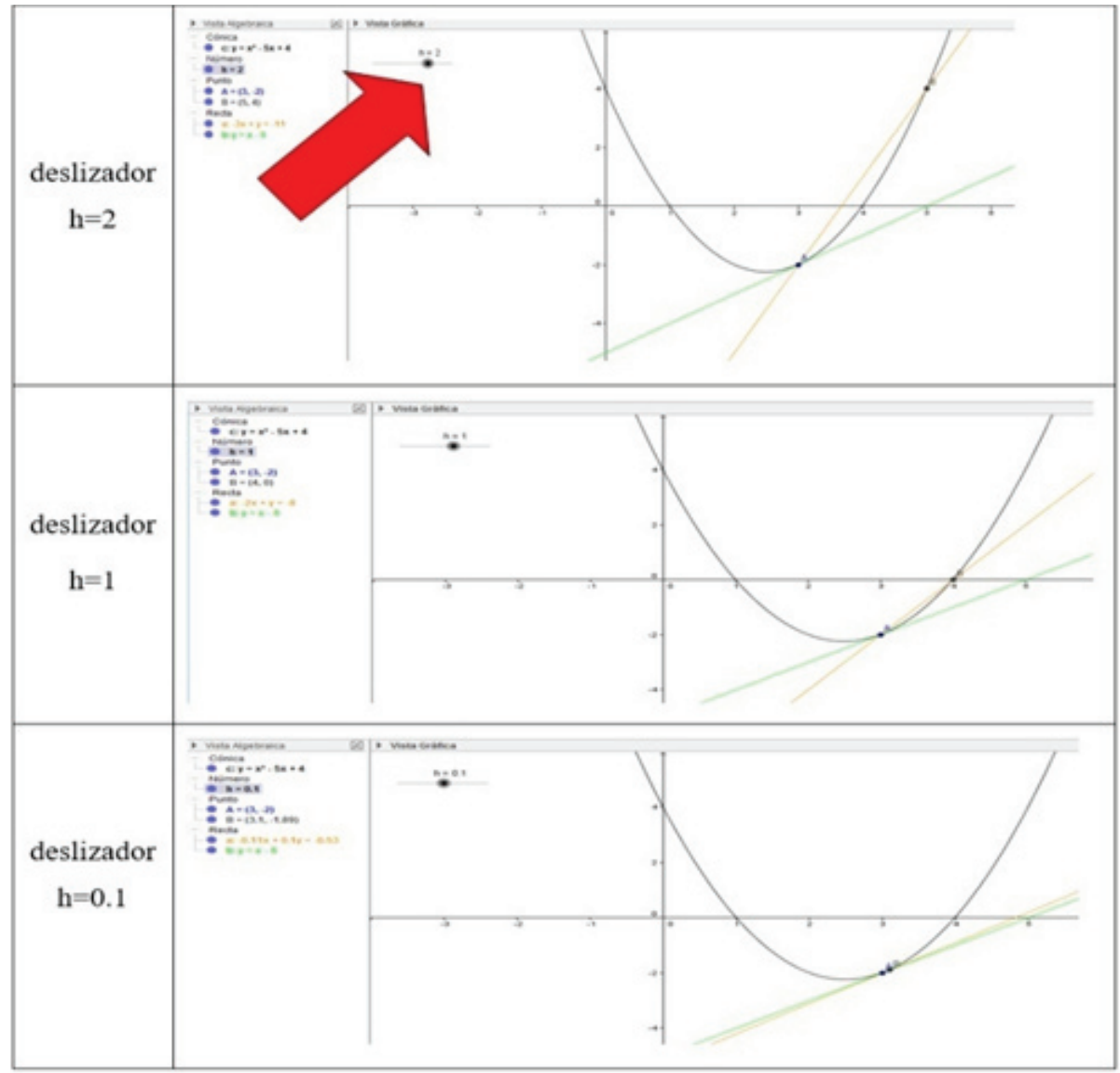

Figura 8. Esquema de acción instrumental que usa un deslizador de GeoGebra para mostrar de manera dinámica la convergencia de las rectas secantes a la recta tangente y permite la transición desde la concepción leibniziana de recta tangente a la concepción cartesiana

\section{DISCUSIÓN Y CONCLUSIONES}

Los resultados de esta investigación nos han permitido identificar tres características de génesis instrumental en la construcción de la concepción cartesiana de la recta tangente en estudiantes de primer curso de educación secundaria pos-obligatoria (16 años). Las tres características del proceso de génesis instrumental identificadas ponen de manifiesto la relación entre el uso de los comandos de GeoGebra y la constitución de esquemas de acción instrumental que usan los significados del concepto y los mecanismos constructivos de conocimiento (tanteo, generalización, coordinación,...). Además, el uso de los comandos 
representación gráfica de una función, el zoom o el cambio de color de la gráfica de una función, entendidos como esquemas de uso, no constituyeron ninguna dificultad para los estudiantes. De esta manera, la evidencia de la existencia de esquemas de acción instrumental la podemos vincular a formas de usar el conocimiento.

Estas características se manifiestan en (i) la relación entre el esquema de uso del comando zoom y la generación de la concepción leibniziana de recta tangente (esquema de acción instrumental); (ii) el uso de los modos de representación y los esquemas de acción instrumental puestos de manifiesto por la mediación semiótica y las aproximaciones por tanteo a la resolución de problemas; y finalmente, (iii) el uso del comando deslizador para generar la coordinación de las concepciones cartesiana y leibniziana del concepto de recta tangente (esquema de acción instrumental). En estas características de los procesos de génesis instrumental generados, los recursos tecnológicos desempeñan un papel fundamental al permitir:

- visualizar la idea de linealidad local de una función en el entorno de un punto en el que la función es derivable (concepción leibniziana) ya que al prolongar el segmento en el que se encuentra el punto de tangencia obtenemos la recta tangente.

- $\quad$ superar las limitaciones de la concepción de recta tangente como aquella que toca la circunferencia pero no la corta (concepción euclídea, válida únicamente para las cónicas).

- coordinar las concepciones cartesiana y leibniziana (visualizando gráficamente de forma dinámica la convergencia de las rectas secantes a la recta tangente previamente definida).

- visualizar la recta tangente como mejor aproximación lineal de una función en el entorno de un punto.

El zoom y el deslizador de GeoGebra han desempeñado un papel crucial en la progresión en el aprendizaje de los estudiantes. Por un lado, el uso del zoom ha favorecido la construcción del significado de la linealidad local de una función (concepción leibniziana). Esto ha permitido definir la recta tangente sin necesidad de realizar un paso al límite como ocurre en la concepción cartesiana. Por otro, el deslizador ha permitido coordinar la concepción cartesiana y la leibniziana. Esta faceta del GeoGebra como mediador ha facilitado el progreso de los estudiantes hacia niveles sofisticados de pensamiento. Cuatro de los cinco grupos de estudiantes en el experimento han interiorizado el concepto de recta tangente pues son capaces de coordinar diferentes registros como el gráfico, el analítico-numérico y el analítico-algebraico.

Como conclusiones podemos destacar tres aspectos. Primero, el enorme potencial del recurso tecnológico (GeoGebra) en el proceso de enseñanza-aprendizaje principalmente a través de la visualización. GeoGebra ha ayudado a transitar desde la concepción euclídea a la concepción cartesiana de recta tangente con la mediación de la concepción leibniziana y mediante la coordinación de los registros gráfico y analítico. La visualización que permite GeoGebra ayudó a los estudiantes a interiorizar la concepción leibniziana con la ayuda del 
zoom (como definición de la recta tangente), a mostrar las limitaciones de la concepción euclídea (aplicable únicamente a las cónicas) o a ver la convergencia dinámica de las rectas secantes a la recta tangente con la ayuda del deslizador.

Segundo, este recurso tecnológico favorece la coordinación de los registros gráfico y analítico y así permite, por un lado, comprobar gráficamente las soluciones obtenidas de forma algebraica o numérica, y por otro, ayuda a que los estudiantes construyan un concepto de recta tangente más completo al permitir relacionar todos los registros: grafico, analítico-algebraico y analítico-numérico. En el experimento de enseñanza se constató que la actividad de los alumnos, en contextos tecnológicos diseñados ad hoc, donde se integran diferentes tipos de representaciones interrelacionadas, ayuda a avanzar en la construcción del concepto de recta tangente. La interacción y el dinamismo facilitaron a los estudiantes la coordinación interna entre las representaciones analíticas y geométricas de este concepto (Duval, 2006). Nuestros resultados están en consonancia con los obtenidos por Maschietto (2008) o Lagrange y Artigue (2009) en el sentido de que los recursos tecnológicos son instrumentos de mediación semiótica gracias a su potencialidad para presentar simultáneamente varias representaciones de un mismo concepto y para favorecer la interacción y el dinamismo. Por otra parte, como señalan Kieran y Drijvers (2006), algunas investigaciones que reúnen el uso de lápiz y papel y los entornos tecnológicos muestran que el valor epistémico de técnicas de lápiz y papel parecen jugar un papel no sólo complementario, sino esencial. Por ello, consideramos que la combinación e integración de ambos tipos de técnicas, tal como se ha hecho en el experimento de enseñanza, ha enriquecido su valor epistémico.

Finalmente, el recurso tecnológico usado ha facilitado el que los estudiantes progresen hacia niveles de pensamiento más sofisticados al permitirles experimentar o comprobar conjeturas que les ayudan a construir un concepto de recta tangente más completo. Por ejemplo, en la actividad 3 de la sesión 4 del experimento de enseñanza en la que se pide obtener la recta tangente a una función paralela a otra recta dada, los estudiantes del grupo 1 saben cuál es la pendiente de la recta tangente por ser paralela a la recta dada, pero desconocen su ordenada en el origen. Para obtenerla van tanteando con diferentes valores y viendo gráficamente qué ocurre con cada uno de ellos hasta llegar a la solución. Esta forma de proceder con lápiz y papel y sin la ayuda del recurso tecnológico sería totalmente inviable. Estos resultados están en línea con las conclusiones obtenidas por Arzarello et al. (2002) y Artigue (2002) relativas a que los recursos tecnológicos permiten a los estudiantes explorar los objetos matemáticos, conjeturar sobre ellos, validar dichas conjeturas y justificar sus resultados.

\section{REFERENCIAS}

Alqahtani, M. y Powell, A. (2016). Instrumental appropriation of a collaborative, dynamicgeometry environment and geometrical understanding. International Journal of Education in Mathematics, Science and Technology, 4(2), 72-83. 
Alqahtani, M. y Powell, A. (2017). Teachers' instrumental genesis and their geometrical understanding in a dynamic geometry environment. Digital Experiences in Mathematics Education, 3(1), 9-38.

Artigue, M. (2002). Learning Mathematics in a CAS environment: the genesis of a reflection about instrumentation and the dialectics between technical and conceptual work. International Journal of Computers for Mathematical Learning, 7(3), 245-274.

Artigue, M. (2007). Tecnología y enseñanza de las matemáticas: desarrollo y aportaciones de la aproximación instrumental Investigación. En: XII Conferencia Interamericana de Educación Matemática México: XII CIAEM, pp. 25-37.

Arzarello, F., Olivero, F., Paola, D. y Robutti, O. (2002). A cognitive analysis of dragging practises in Cabri environments. International Reviews on Mathematical Education (ZDM), 34(3), 66-72.

Boigues, F. J., Estruch, V. y Llinares, S. (2010). El papel de sistemas de cálculo formal en la comprensión de las matemáticas: el caso de la integral definida. Modeling in Science Education and learning, 3(1), 3-18.

Drijvers, P. y Trouche, L. (2008). From artifacts to instruments. Research on technology and teaching and learning of mathematics, 2, 363-391

Drijvers, P, Kieran, C. y Mariotti, M.A. (2010). Integrating technology into mathematics education: Theoretical perspectives. En C. Hoyles y J.B. Lagrange (Eds.), Mathematics education and technology: Rethinking the terrain (pp. 89-132). New York: Springer.

Duval, R (2006). A cognitive analysis of problems of comprehension in a learning of mathematics. Educational Studies in Mathematics, 61, 103-131.

Faggiano, E., Ferrara, F. y Montone, A. (Eds.) (2017). Innovation and Technology Enhancing Mathematics Education. London: Springer.

Guin, D. y Trouche, L. (1999). The Complex Process of Converting Tools into Mathematical Instruments. The Case of Calculators. International Journal of Computers for Mathematical Learning, 3(3), 195-227.

Kieran, C. y Drijvers, P. (2006). The co-emergence of machine techniques, paper-andpencil techniques, and theoretical reflection: a study of CAS use in secondary school algebra. International Journal of Computers for Mathematical Learning, 11, 205-263.

Lagrange, J. B. y Artigue, M. (2009). Student's activities about functions at upper secondary level: A grid for designing a digital environment and analyzing uses. En M. Tzekaki, M. Kaldrimidou y H. Sakonidis (Eds.), Proceedings of the $33^{\text {rd }}$ Conference of the International Group for the Psychology of Mathematics Education (vol.3, pp.465472). Grecia: PME.

Maschietto, M. (2008). Graphic Calculators and micro-straightness: Analysis of a didactic engineering. International Journal of Computers for Mathematical Learning, $13,207-230$.

NCTM (2015). Strategic Use of Techonology in Teaching and Learning Mathematics. A Position of the National Council of Teacher Mathematics. Recuperado el 10 de enero de 2018 de http://www.nctm.org/Standards-and-Positions/Position-Statements/StrategicUse-of-Technology-in-Teaching-and-Learning-Mathematics/ 
Orts, A. (2017). Características del aprendizaje de la recta tangente. Identificación de trayectorias de aprendizaje en un experimento de enseñanza. Tesis doctoral. Departamento de Innovación y Formación Didáctica. Universidad de Alicante.

Orts, A., Llinares, S. y Boigues, F.J. (2016a). El papel de los recursos tecnológicos en el aprendizaje de matemáticas en estudiantes de Bachillerato. En R. Roig-Vila (Ed.), Tecnología, innovación e investigación en los procesos de enseñanza-aprendizaje (pp. 1002-1011). Barcelona: Octaedro.

Orts, A., Llinares, S. y Boigues, F.J. (2016b). Elementos para una Descomposición Genética del concepto recta tangente. Avances de Investigación en Educación Matemática, 10, 111-134.

Rabardel, P. (1995). Les hommes et les technologies: approche cognitive des instruments contemporains. Paris: Armand Colin.

Rabardel, P. y Beguin, P. (2005). Instrument mediated activity: from subject development to anthropocentric design. Theoretical Issues in Ergonomics Science, 6(5), 429-461.

Stahl, G. (2015). Constructing dynamic triangles together: The development of mathematical group cognition. Cambridge, Reino Unido: Cambridge.

Trouche, L. (2004). Managing the Complexity of Human/Machine Interactions Environments: Guiding Student's Command Process Through Instrumental Orchestrations. International Journal of Computers for Mathematical Learning, 9(3), 281-307.

Verillon, P. y Rabardel, P. (1995). Cognition and artifacts: A contribution to the study of thought in relation to instrument activity. European Journal of psychology of education, $9(3), 77-101$. 